\title{
Characterization of Titanium Dioxide Pigment Particles by High-Resolution and Analytical Transmission Electron Microscopy (HRTEM and AEM)
}

Wei Wayne ZHAO

Tronox LLC, Technical Center, 3301 NW 150 ${ }^{\text {th }}$ St., Oklahoma City, OK 73134 USA

Rutile titanium dioxide $\left(\mathrm{TiO}_{2}\right)$ surpasses performance of other types of white pigments in applications such as coatings, papers, plastics, and inks [1]. The combination of high refractive index, durability, cost-effectiveness, and environmental friendliness are important commercial features of this pigment. For optimum optical performance, the average diameter of the $\mathrm{TiO}_{2}$ particles should be around 200-300 nanometers. The $\mathrm{TiO}_{2}$ particles are commonly surface modified with inorganic and organic coatings to improve performance in the application. For example, dense silica coating is used to enhance pigment durability and alumina coating is added to improve dispersibility in coatings [2].

The unique spatial resolution of atomic-scale high-resolution transmission electron microscopy (HRTEM) and analytical scanning transmission electron microscopy (AEM) plays a critical role in understanding the nature and performance of pigments and their surface coatings. Provided here are preliminary studies showing the application of HRTEM and AEM in research and development, process engineering, and physical failure analysis for several experimental $\mathrm{TiO}_{2}$ samples.

Figure 1 is an example of crystallinity and crystal defect investigations using HRTEM lattice imaging. Understanding the crystal growth habit facilitates the design and optimization of process parameters, e.g., to control degree of rutilization, to control nucleation rate and to tailor crystal growth for optimal particle size. In-depth understandings of the integrity of the surface coatings help to optimize performance of the pigment in the end use application. The uniformity and optimal thickness are keys to achieving the full functionality of these surface coatings (Fig 2).

In support of product and process development, HRTEM (Fig 3) and AEM along with x-ray energy dispersive spectroscopy (XEDS) and associated elemental linescan (Fig. 4) help to elucidate the chemical nature of the interfacial materials. Another analytical component of AEM, electron energy loss spectroscopy (EELS), can also be applied only if the nanoparticles can be successfully sectioned into "perfectly flat" TEM foil, since EELS is a thickness-sensitive technique. It is an ongoing challenge to slice ceramic-like $\mathrm{TiO}_{2}$ particles without damaging the pigment surface coatings, such as introduction of artifacts resulting from focused ion beam (FIB) bombardment.

\section{References}

[1] J. H. Braun, White Pigment, Federation of Societies for Coatings Technology, Blue Bell, PA, USA, 1995.

[2] T. A. Egerton, KONA Powder and Particle, 16 (1998) 46 47.

[3] The aid from Dr. Michael Greene for review the manuscript is gratefully acknowledged.

[4] The support from Legal and Management teams of Tronox is appreciated, www.tronox.com.

[5] Thanks also go to Barbara Woodbury and Collette Duncan for the TEM sample-prep. 


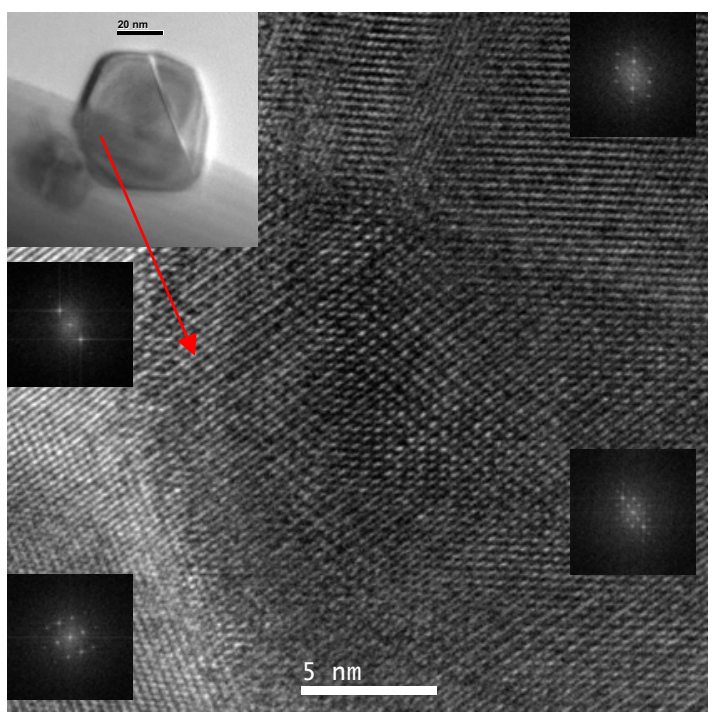

Fig. 1. HRTEM lattice image on an experimental sample discerned the co-growth of the $\mathrm{TiO}_{2}$ crystals.

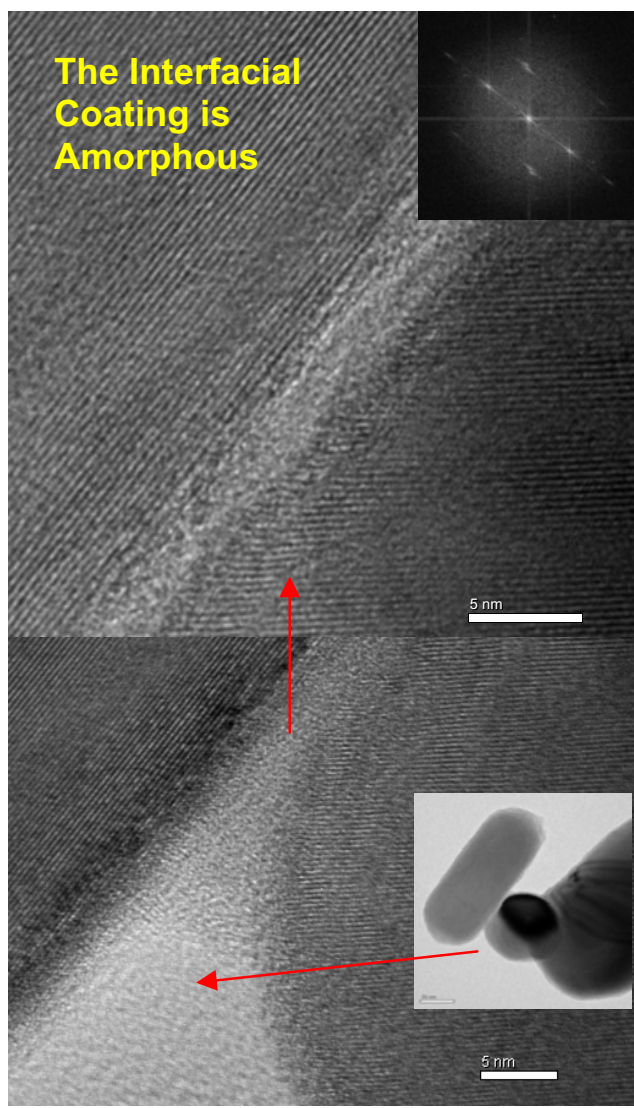

Fig. 3. HRTEM on an experimental $\mathrm{TiO}_{2}$ agglomeration with amorphous interfacial coatings.

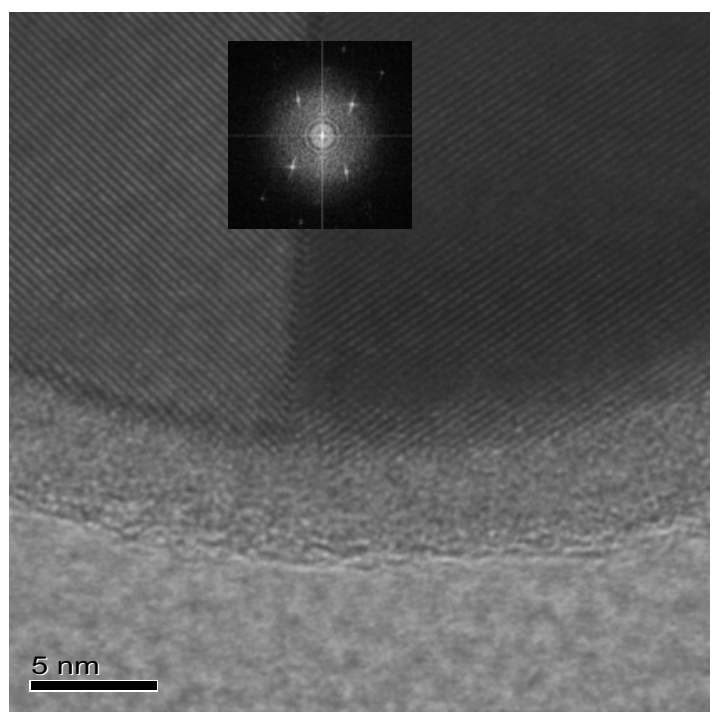

Fig. 2. HRTEM on an experimental $\mathrm{TiO}_{2}$ sample with surface coatings.

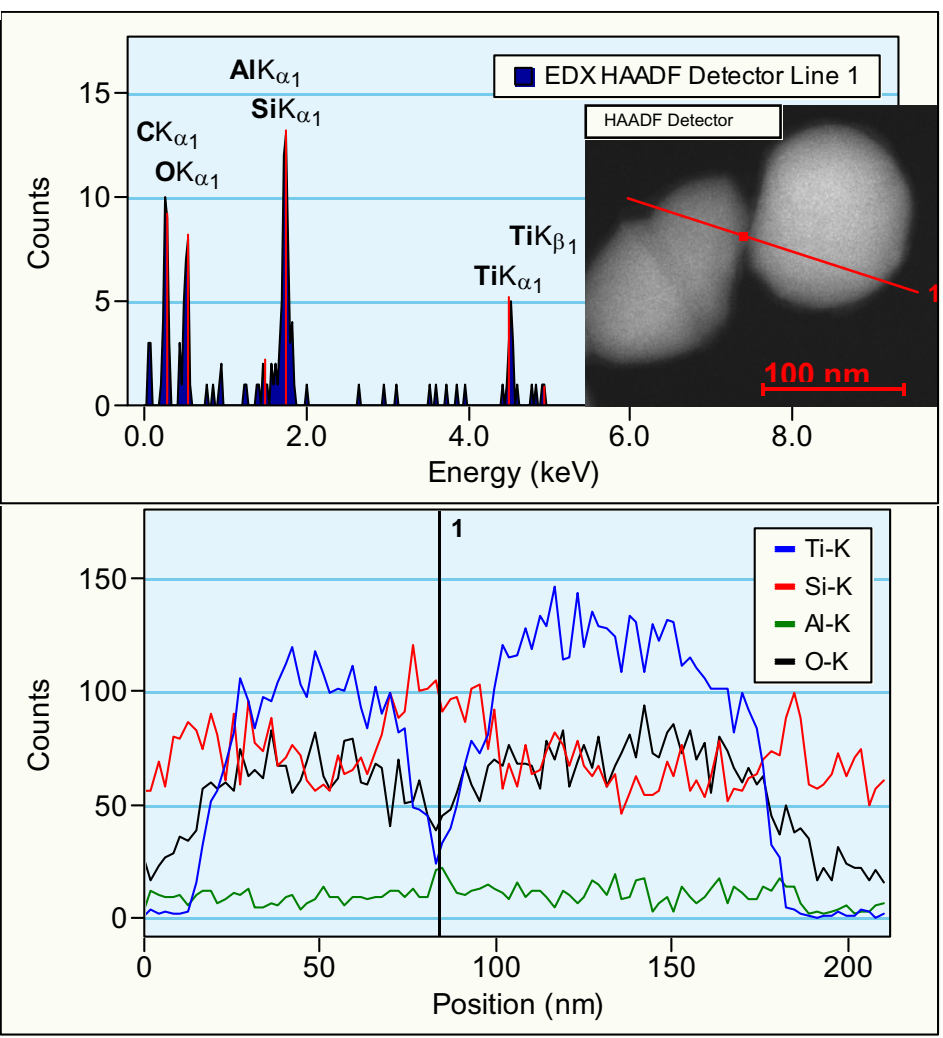

Fig. 4. HAADF-STEM imaging with X-ray EDS spectrum and associated elemental linescan indicated the chemical profiles along the interface of agglomeration from an experimental $\mathrm{TiO}_{2}$ sample for a durable grade pigment. 